\title{
School scoliosis screening in Hong Kong: trunk asymmetry of girls with scoliosis
}

\begin{abstract}
Background: The school scoliosis screening aims to identify students with spinal deviations by trunk asymmetry. The objective of this study is to investigate the accuracy of scoliosis screening with a low ATR cut-off and occurrence of shoulder obliquity.
\end{abstract}

Methods: 443 schoolgirls aged between 10-13 years participated in this school scoliosis screening. During the screening, a forward bending test with ATR measurement using scoliometer were adopted to detect the trunk asymmetry and the backside photographs were captured for shoulder obliquity measurement. The ATR cut-off point was $3^{\circ}$. Participants with ATR $\geq 3^{\circ}$ were invited to conduct an ultrasound spinal examination for Cobb's angle measurement. The positive predictive value of $A T R \geq 3^{\circ}$ was calculated.

Findings: It is found that $17.8 \%$ of the screened students possibly have early scoliosis. The positive predictive value of ATR $\geq 3^{\circ}$ was 0.717 . There is a significant negative correlation between the BMI range and occurrence of ATR $\geq 3^{\circ} .55 .7 \%$ of girls with $\mathrm{ATR} \geq 3^{\circ}$ had shoulder obliquity. There is a significant positive correlation between the occurrence of shoulder obliquity and ATR $\geq 3^{\circ}$.
Volume I2 Issue I - 2020

\author{
Queenie Fok,' Pak-Yiu Liu,' Joanne Yip,' Mei- \\ chun Cheung,' Kit-Lun Yick,' Sun-Pui Ng,, \\ Chi-Yung Tse ${ }^{4}$ \\ 'Institute of Textiles and Clothing, The Hong Kong Polytechnic \\ University, Hong Kong \\ ${ }^{2}$ Department of Social Work, The Chinese University of Hong \\ Kong, Hong Kong \\ ${ }^{3}$ Hong Kong Community College, The Hong Kong Polytechnic \\ University, Hong Kong \\ ${ }^{4}$ Centre for Orthopaedic Surgery, Bank of America Tower, \\ Central, Hong Kong
} Correspondence: Joanne Yip, Institute of Textiles and Clothing,
The Hong Kong Polytechnic University, Hung Hom, Hong Kong,
Email tcjyip@polyu.edu.hk

Received: December 20, 2019 | Published: January 17, 2020

\section{Introduction}

Adolescent Idiopathic scoliosis (AIS) is a spinal condition with curvature equal or greater than 10 degrees which is commonly onset during puberty with undefined etiology. The prevalence rate of AIS was $0.38-7.45 \%$ and the girls-to-boys ratio was $2.7-5.14 .{ }^{1-3}$ Scoliosis would cause the trunk asymmetry, induce spinal spin, restrict body movement or even deteriorate the cardiac and pulmonary functions. ${ }^{4}$ Nevertheless, early detection and subsequent treatment such as bracing, physical therapy, surface electrical stimulation, and chiropractic could prevent the curve progression and preclude the need of surgery. ${ }^{2}$

Postural alterations are commonly found in the adolescents with AIS including the orientation of the head, shoulders, scapular, and pelvis in all three planes, while rotations of body segments in the horizontal plane are more particular. So, AIS can be noticed by the postural "warning signs", including curved-body, rib prominence at one side, flank prominence at one side, curved head-rib-pelvis line, one side of the pelvis tilt up, head is not at the direct center of above the pelvis, shoulder blade prominence at one side, scapula rotated, raised and prominent uneven hip. ${ }^{5}$

School scoliosis screening aims to detect back trunk asymmetry in children at risk to develop progressive scoliosis and provides valuable knowledge about prevalence, etiology and natural history of AIS. ${ }^{6}$ It also contributes to the evaluation of health needs of a population and allocation of health resources.

School screening for AIS has been performed in certain countries for many years. ${ }^{7}$ In Hong Kong, school screening for AIS consists of a voluntary 3-tier assessment provided by Department of Health, the Government of the Hong Kong Special Administrative Region has been implemented since 1995 . The screening program is conducted for students in Primary 5, Secondary 1 and 3 (equivalent to age 10, 12 , and 14 , respectively). They will be first screened with the forward bending test and scoliometer is used to measure the angle of trunk rotation (ATR). Students are rescreened annually if they have an ATR of $3^{\circ}$ or $4^{\circ}$. If the ATR lay between $5^{\circ}$ and $14^{\circ}$, they will be submitted to second-tier assessment by moiré topography. If they have either an ATR of $15^{\circ}$ or above, or 2 or more moiré lines, they will be referred for radiography. ${ }^{2}$

The effectiveness of school scoliosis screening is determined by the screening test and screening age. Forward bending test with ATR measurement is a classic screening test for scoliosis. However, the cut-off of ATR is still controversial. ${ }^{1}$ A high cut-off could screen a scoliosis with a high positive predictive rate, but it may result a low sensitivity due to a high rate of omission. Luk et al. ${ }^{2}$ studied the clinical effectiveness of school screening for AIS and reported that the sensitive of curvature $\geq 10^{\circ}$ for adolescent with ATR $\geq 15^{\circ}$ was $64.7 \%$ only. It may implicate that over one third of cases were omitted when the cut-off ATR is $15^{\circ}$.

The objective of this study is to investigate the accuracy of school scoliosis program with a low ATR cut-off and the occurrence of trunk asymmetry of female students in Hong Kong.

\section{Materials and methods}

\section{Study design}

This cross-sectional study was performed between September 2011 and August 2013 in 2 secondary schools and 4 primary schools in Hong Kong. In total, 443 schoolgirls were screened. The study was approved by The Hong Kong Polytechnic University and informed consent was obtained from the participants and their parents. 


\section{Preparation for school screening}

Before screening, participants were asked to remove their outerwear and shoes. Since students at the puberty stage were selfconscious, to lessen their anxieties, provide a comfortable screening environment, and protect their privacy, all participants were screened individually, in private, accompanied by female researchers and were encouraged to wear sports bra. Moreover, all windows were covered and a private area for changing clothing near the examination area were arranged. ${ }^{5}$

In addition, in order to collect the backside photographs for shoulder obliquity measurement, an area was reserved for photo taking. A sheet with $5 \mathrm{~cm} \times 5 \mathrm{~cm}$ metric grids which acted as reference lines was set on a wall. A digital camera was rotated and locked in the vertical position in order to capture the entire body. The camera was positioned on a tripod at a standard distance of $2 \mathrm{~m}$ away from the participants and at a height of $1 \mathrm{~m} .^{8}$

\section{School screening program}

Demographic characteristics including age, weight, height, clinical photo images of back and results of screening which is the ATR were collected and documented for each participant.

The screening procedure combined the forward bending test and scoliometer measurement of ATR. ${ }^{6}$ A prosthetist-orthotist ( $\left.\mathrm{P} \& \mathrm{O}\right)$ was engaged as a screener. During the forward bending test, participants were asked to place the chin to the chest, put hands together, and bend forward from the waist 90 degrees slowly. ${ }^{5}$ Then, the screener measured the ATR in the thoracic and lumbar prominence with a scoliometer (OSI, Orthopaedic Systems Inc., Hayward, California, USA). Three degrees of ATR was chosen as cut-off for suspected case of scoliosis. ATR $<3^{\circ}$ are considered as normal while ATR $\geq 3^{\circ}$ as sign of early scoliosis.

Participants with a positive screening result were invited to under an ultrasound spinal examination. Scolioscan (Telefield Mdical Imaging Limited, Hong Hong) was used to acquire the ultrasound spinal image for the Cobb's angle measurement. ${ }^{9}$ A Cobb's angle $\geq 10^{\circ}$ on standing were classified as AIS according to the criteria proposed by the Scoliosis Research Society. ${ }^{10}$

\section{Measurement of shoulder obliquity on digital photographs}

CorelDraw (Corel Corporation, Ontario, Canada) was used for shoulder obliquity measurement on digital photographs. The shoulder obliquity was measured by the clavicle angle (CA) defined by the angle between the intersecting of a line of the two highest points on the clavicle and a line that is the horizontal reference line marked (Figure 1). A positive value was assigned when the left side was higher than the right side, and a negative value was assigned when the right side was higher than the left side. The cut-off point of the CA was set as $\pm 1^{\circ}$. CA with $0 \pm 1^{\circ}$ was considered as shoulder levelness that is neutral while $\mathrm{CA} \geq 1^{\circ}$ or $\mathrm{CA} \leq-1^{\circ}$ was considered as the presence of shoulder obliquity. ${ }^{11}$

\section{Statistical analysis}

Occurrence of suspected scoliosis was calculated by number of participants with ATR $\geq 3 \%$ number of total participants $x 100 \%$. The positive predictive value was calculated by number of participants with Cobb's angle $\geq 3 \%$ number of participants conducted ultrasound spinal examination. Occurrence of shoulder obliquity was calculated by number of participants with $\mathrm{CA} \geq 1^{\circ}$ or $\mathrm{CA} \leq-1^{\circ} /$ number of total participants $\mathrm{x} 100 \%$. Body mass index (BMI) was calculated by weight $/$ height $^{2}\left(\mathrm{~kg} / \mathrm{m}^{2}\right)$. BMI $<18.5$ are considered as underweight, between $18.5-24.9$ as normal and $>25$ as overweight.

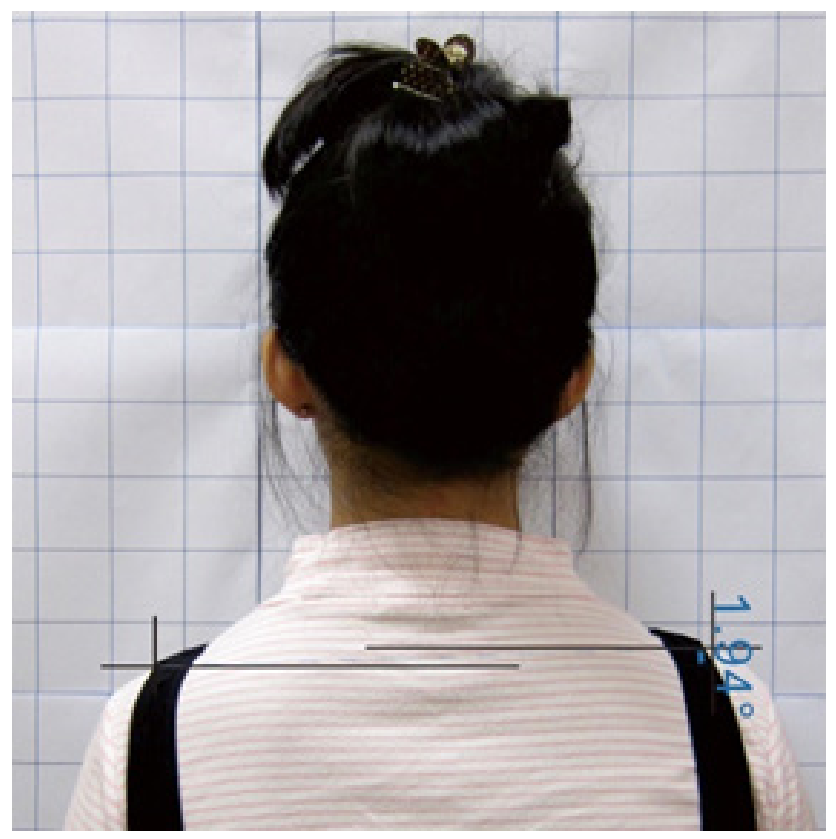

Figure I Measuring method of CA on clinical photo in CorelDraw.

Non-parametric Spearman's rank order correlation was performed to investigate the correlation between the variables. The statistical Package for the Social Sciences, version 19.0 (SPSS Inc., Chicago, USA) was used and the confidence interval was set at $95 \%(\mathrm{P}<0.05)$.

\section{Results and discussion}

In this study, screening has been carried out at 2 secondary schools and 4 primary schools, in order to investigate the spinal conditions of the targeted group. A total of 443 females aged 10-13 years old were screened by a P\&O.

It was found that $79(17.8 \%)$ females out of the 443 screened participants may have early scoliosis (with ATR $\geq 3^{\circ}$ ). ATR readings are obtained in the form of a non-negative integer number in this study. The screening result in previous studies however are difficult to comparable since the age group, gender, and cut-off point varied across studies. ${ }^{1}$ Wang et al. ${ }^{12}$ conducted a scoliosis screening in Beijing of children aged between 8-14 years old and reported that occurrence of ATR $\geq 3^{\circ}$ was $4.15 \%$. Adobor et al. ${ }^{6}$ mentioned the screening results in Netherlands that the occurrence of ATR $\geq 7^{\circ}$ for children aged between 12 was $1.5 \%$.

Of 79 participants with ATR $\geq 3^{\circ}, 60$ underwent the ultrasound spinal examination and 43 have a positive result with a Cobb's angle $\geq 10^{\circ}$. The positive predictive value of screening with ATR $\geq 3^{\circ}$ was 0.717 . The positive predictive value largely varied across study since it is inversely related to the degree of Cobb's angle adopted to define scoliosis and the cut-off of ATR. Fong et al. ${ }^{1}$ conducted a metaanalysis cited 34 studies about school scoliosis screening and pooled estimated positive predictive value for Cobb's angle $\geq 10^{\circ}$ were 0.28 . Luk et al. ${ }^{2}$ mentioned that the positive predictive value of ATR $\geq 15^{\circ}$ 
for Cobb's angle $\geq 10^{\circ}$ was 0.765 . It implicated the considering $3^{\circ}$ of ATR as cut-off point might lower positive predictive value. The advantage of low cut-off point is the high sensitivity.

In the school screening program of this study, participants who have an ATRI $\geq 3^{\circ}$ were treated as suspected cases in order to screen out those who have "early scoliosis". However, in the screening program carried out by the Department of Health, sometimes the participants would only be considered to a radiographic examination if they had an ATR $\geq 15^{\circ}$. The effectiveness of screening program might be doubtful since some early scoliosis case may be screened out due to the high cut-off point. Burwell et al. ${ }^{13}$ hypothesized the vicious cycle of scoliosis progression pointing out that the wedged vertebrae in early scoliosis results in continuous asymmetric loading on the spine and perpetuate the asymmetric growth of spine. Early detection for scoliosis and treatment for early scoliosis could avoid or decelerate the progression. ${ }^{14}$ Thus, a low cut-off point might be recommended to detect the early scoliosis.

The age and BMI distributions of the screened participants are summarized in Table 1. The participants were assigned to the normal subject group ( $\mathrm{N}$ group) if they have an ATR $0-2^{\circ}$ and to the suspected scoliosis group ( $\mathrm{S}$ group) if they have an ATIR $\geq 3^{\circ}$. From Table 1, it was surprising that $75.9 \%$ scoliosis suspected participants were underweight. A Spearman's rank-order correlation was run to determine the relationship between the BMI range and occurrence of suspected scoliosis. There was a significant negative monotonic correlation between the BMI range and occurrence of suspected scoliosis ( $r=-0.95, n=443, p=0.046)$. It indicated that underweight adolescent has a relative high probability of scoliosis.

Table I Age and BMI distributions of screened participants

\begin{tabular}{|c|c|c|c|c|c|c|c|c|}
\hline \multirow{2}{*}{$\begin{array}{l}\text { Age } \\
10\end{array}$} & \multirow{2}{*}{$\begin{array}{l}\text { Group } \\
\mathrm{N}\end{array}$} & \multicolumn{2}{|c|}{ Underweight } & \multicolumn{2}{|c|}{ Normal weight } & \multicolumn{2}{|c|}{ Overweight } & \multirow{2}{*}{\begin{tabular}{|l} 
Total \\
68
\end{tabular}} \\
\hline & & 44 & $64.70 \%$ & 21 & $30.90 \%$ & 3 & $4.40 \%$ & \\
\hline & & $84.60 \%$ & & $95.50 \%$ & & $75 \%$ & & $87.20 \%$ \\
\hline & S & 8 & $80 \%$ & 1 & $10 \%$ & I & $10 \%$ & 10 \\
\hline & & $15.40 \%$ & & $45.50 \%$ & & $25 \%$ & & $7.80 \%$ \\
\hline & Total & 52 & $66.70 \%$ & 22 & $28.20 \%$ & 4 & $5.10 \%$ & 78 \\
\hline \multirow[t]{5}{*}{ II } & $\mathrm{N}$ & 102 & $69.90 \%$ & 32 & $21.90 \%$ & 12 & $8.20 \%$ & 146 \\
\hline & & $77.90 \%$ & & $80 \%$ & & $92.30 \%$ & & $79.30 \%$ \\
\hline & $S$ & 29 & $76.30 \%$ & 8 & $21.10 \%$ & I & $2.60 \%$ & 38 \\
\hline & & $22.10 \%$ & & $20 \%$ & & $7.70 \%$ & & $20.70 \%$ \\
\hline & Total & $13 \mid$ & $71.20 \%$ & 40 & $21.70 \%$ & 13 & $7.10 \%$ & 184 \\
\hline \multirow[t]{5}{*}{12} & $\mathrm{~N}$ & 79 & $58.90 \%$ & 47 & $35.10 \%$ & 8 & $6.00 \%$ & 134 \\
\hline & & $79.80 \%$ & & $88.70 \%$ & & $88.90 \%$ & & $83.20 \%$ \\
\hline & S & 20 & $74.10 \%$ & 6 & $22.20 \%$ & 1 & $3.70 \%$ & 27 \\
\hline & & $20.20 \%$ & & $11.30 \%$ & & $11.10 \%$ & & $16.80 \%$ \\
\hline & Total & 99 & $61.50 \%$ & 53 & $32.90 \%$ & 9 & $5.60 \%$ & 161 \\
\hline \multirow[t]{5}{*}{13} & $\mathrm{~N}$ & 7 & $43.80 \%$ & 6 & $37.50 \%$ & 3 & $18.70 \%$ & 16 \\
\hline & & $70 \%$ & & $85.70 \%$ & & $100 \%$ & & $80 \%$ \\
\hline & S & 3 & $7.50 \%$ & 1 & $25 \%$ & 0 & $0 \%$ & 4 \\
\hline & & $30 \%$ & & $14.30 \%$ & & $0 \%$ & & $20 \%$ \\
\hline & Total & 10 & $50 \%$ & 7 & $35 \%$ & 3 & $15 \%$ & 20 \\
\hline \multirow[t]{5}{*}{ All age } & $N$ & 232 & $63.80 \%$ & 106 & $29.10 \%$ & 26 & $7.10 \%$ & 364 \\
\hline & & $79.50 \%$ & & $86.90 \%$ & & $89.70 \%$ & & $82.20 \%$ \\
\hline & $S$ & 60 & $75.90 \%$ & 16 & $20.30 \%$ & 3 & $3.80 \%$ & 79 \\
\hline & & $20.50 \%$ & & $13.10 \%$ & & $10.30 \%$ & & $17.80 \%$ \\
\hline & Total & 292 & $65.90 \%$ & 122 & $27.50 \%$ & 29 & $6.60 \%$ & 443 \\
\hline
\end{tabular}

*Remarks: N, normal group (participants with ATI 0-2 ${ }^{\circ}$ ); S, suspected group (participants with ATI $\geq 3^{\circ}$ ) 
A Spearman's rank-order correlation was run to determine the relationship between the age and occurrence of suspected scoliosis. There was no significant correlation between the age and occurrence of suspected scoliosis $(\mathrm{p}=0.717$ ). Children aged between 10 and 14 years are most likely to develop scoliosis. ${ }^{1}$ The current results further confirmed that there is a need to conduct scoliosis screening for age group between 10 and 13 years.

In this study, shoulder obliquity was found in $19.8 \%$ of girls in normal group and $55.7 \%$ of girls in suspected scoliosis group. The overall occurrence of shoulder obliquity was $26.2 \%$. It indicated that the occurrence of shoulder obliquity in both groups is high and it raised the attention on adolescent posture problems. The current result agrees with a previous study done by Sekouris \& Smyrnis ${ }^{15}$ which pointed out that the $31 \%$ of people without scoliosis have shoulder asymmetry. It implicated that aside from scoliosis, there are some other possible factor of shoulder obliquity.

A Spearman's rank-order correlation was run to determine the relationship between the occurrence of shoulder obliquity and suspected scoliosis. There was a significant positive monotonic correlation between the occurrence of shoulder obliquity and suspected scoliosis $(\mathrm{rs}=0.401, \mathrm{n}=443, \mathrm{p}<0.001)$. This result could be explained by the concordance between the proximal end of thoracic spine (T1) and shoulder. The orientation of shoulder is dependent on the proximal and main thoracic curve. ${ }^{16}$ Nevertheless, results of Spearman's rank-order correlation indicated that there was no significant correlation between the occurrence of shoulder obliquity and BMI range $(\mathrm{p}=0.098)$ and between the occurrence of shoulder obliquity and age $(\mathrm{p}=0.209)$.

\section{Conclusion}

A school screening program with a cut-off point of ATR of $3^{\circ}$ was carried out in Hong Kong during 2011 to 2013 and the target population was 10-13 year-old females. It was found that $17.8 \%$ of participants were suspected to have early scoliosis (with an ATR $\geq 3^{\circ}$ ) and the positive predictive value was 0.717 . A strong negative correlation was found between BMI range and occurrence of suspected scoliosis but there was no significant correlation between the age and occurrence of suspected scoliosis. Moreover, $55.7 \%$ of suspected scoliosis participants had the should obliquity. There was a significant positive correlation between the occurrence of shoulder obliquity and suspected scoliosis, but the occurrence of shoulder obliquity was not significantly correlated with BMI range and age.

\section{Acknowledgments}

The work is supported by funding from PolyU Strategic Development funding scheme [ZVNN] entitled "Posture Correction Girdle for Patients with Idiopathic Scoliosis" and a research studentship granted to Ms. Fok Lai Hing (RULA) from The Hong Kong Polytechnic University.

\section{Conflicts of interest}

The authors declare there are no conflicts of interest.

\section{References}

1. Fong DY, Lee CF, Cheung KM, et al. A meta-analysis of the clinical effectiveness of school scoliosis screening. Spine. 2010;35(10):10611071 .

2. Luk KD, Lee CF, Cheung KM, et al. Clinical effectiveness of school screening for adolescent idiopathic scoliosis: A large population-based retrospective cohort study. Spine (Phila Pa 1976). 2010;35(17):16071614.

3. Hengwei F, Zifang H, Qifei W, et al. Prevalence of idiopathic scoliosis in chinese schoolchildren: a large, population-based study. Spine. 2016;41(3):259-264.

4. McElvenny RT. Structural scoliosis. Clin orthop. 1953;1:87-98.

5. Standards for scoliosis screening in California public schools. California Department of Education: Sacramento. 2007

6. Adobor RD, Rimeslatten S, Steen H, et al. School screening and point prevalence of adolescent idiopathic scoliosis in 4000 Norwegian children aged 12 years. Scoliosis. 2011;6:23-23.

7. Horne JP, Flannery R, Usman S. Adolescent idiopathic scoliosis: diagnosis and management. Am Fam Physician. 2014;89(3):193-198.

8. Canales JZ, Cordás TA, Fiquer JT, et al. Posture and body image in individuals with major depressive disorder: a controlled study. Braz J Psychiatry. 2010;32(4):375-380.

9. Zheng R, Hill D, Hedden D, et al. Assessment of curve progression on children with idiopathic scoliosis using ultrasound imaging method. Eur Spine J. 2018;27(9):2114-2119.

10. Negrini S, Donzelli S, Aulisa AG, et al. 2011 SOSORT guidelines: Orthopaedic and Rehabilitation treatment of idiopathic scoliosis during growth. Scoliosis Spinal Disord. 2018;13:3.

11. Kuklo RT, Lenke LG, Graham EJ, et al. Correlation of radiographic, clinical, and patient assessment of shoulder balance following fusion versus nonfusion of the proximal thoracic curve in adolescent idiopathic scoliosis. Spine. 2002;27(18):2013-2020.

12. Wang YP, Ye QB, Wu B. Result on the screening of scoliosis among school students in Beijing area. Zhonghua Liu Xing Bing Xue Za Zhi. 1996;17(3):160-162.

13. Stokes IA, Burwell RG, Dangerfield PH, et al. Biomechanical spinal growth modulation and progressive adolescent scoliosis - a test of the 'vicious cycle' pathogenetic hypothesis: Summary of an electronic focus group debate of the IBSE. Scoliosis. 2006;1:16.

14. Berdishevsky, H, Lebel VA, Bettany-Saltikov J, et al. Physiotherapy scoliosis-specific exercises - a comprehensive review of seven major schools. Scoliosis Spinal Disord. 2016;11:20.

15. Sekouris N, Smyrnis P. First rib asymmetry and shoulder imbalance assessment of first rib index (FRI) in thoracic X-rays of people without scoliosis. Scoliosis. 2009;4(S1).

16. Menon KV, Tahasildar N, Pillay HM, et al. Patterns of shoulder imbalance in adolescent idiopathic scoliosis: a retrospective observational study. $J$ Spinal Disord Tech. 2014;27(7):401-408. 